CONTROLLING UND MANAGEMENT

Evelyn Raths

Restwertermittlung in der Unternehmensbewertung 
Die Reihe "Controlling und Management" wird herausgegeben von

Prof. Dr. Stefan Dierkes, Georg-August-Universität Göttingen Prof. Dr. Gunther Friedl, Technische Universität München Prof. Dr. Burkhard Pedell, Universität Stuttgart

Band 19 
Evelyn Raths

\section{Restwertermittlung in der Unternehmensbewertung}


Die Deutsche Nationalbibliothek verzeichnet diese Publikation in der Deutschen Nationalbibliografie; detaillierte bibliografische Daten sind im Internet über http://dnb.d-nb.de abrufbar.

Zugl.: Göttingen, Univ., Diss., 2018

ISBN 978-3-8487-5040-5 (Print)

ISBN 978-3-8452-9212-0 (ePDF)

1. Auflage 2019

(c) Nomos Verlagsgesellschaft, Baden-Baden 2019. Gedruckt in Deutschland. Alle Rechte, auch die des Nachdrucks von Auszügen, der fotomechanischen Wiedergabe und der Übersetzung, vorbehalten. Gedruckt auf alterungsbeständigem Papier. 
The value of an instant.

The fascination of independence.

The blessing of an attention to detail.

The ambition to go further

- sensing a pure delight in growing and being oneself.

The value of memories.

The strength of moments that will live in my mind forever

- values not quantifiable with any mathematical formulas.

These invaluable assets and their most worthwhile net investments, an appreciated return on personal investment and mutual benefit in shaping a great future

- fundamentals worth struggling for.

Written with richness of detail.

Dedicated to a strong, respectful, conscious life

- with an infinitely high Continuing Value. 
https://doi.org/10.5771/9783845292120-1

Generiert durch IP '172.22.53.54', am 26.04.2023, 14:15:54.

Das Erstellen und Weitergeben von Kopien dieses PDFs ist nicht zulässig. 
Inhaltsübersicht

1. Einleitung 41

1.1 Problemstellungen und Zielsetzungen 41

1.2 Gang der Untersuchung 50

I. Theoretische Grundlagen 53

2. Theoretische Grundlagen der kapitalmarktorientierten Unternehmensbewertung

2.1 Bewertungsmethodische, konzeptionelle und steuerliche Grundlagen

2.2 Finanzierungstheoretische Grundlagen und Bestimmung von Kapitalkostensätzen

2.3 Bewertung mittels des Free Cashflow (FCF) und des Flow to Equity (FtE) Verfahrens sowie des Residualgewinn-Ansatzes

2.4 Determinanten des Wachstums in der Restwertphase und Marktwertimplikationen

II. Vor- und Nachsteuerrechnung

3. Restwertermittlung in einer Vorsteuerrechnung

3.1 Kritische Reflexion von Vorsteuermodellen und Erweiterungspotenziale für die Restwertmodellierung

3.2 Konzeption, Aufbau und Anwendung eines phasen- und geschäftsbereichsdifferenzierenden Vorsteuermodells

3.3 Konzeption, Aufbau und Anwendung der Restwertermittlung bei gewinnorientierter Ausschüttung im eingeschwungenen Zustand

3.4 Würdigung des erweiterten Vorsteuermodells aus theoretischer und bewertungspraktischer Sicht 
4. Restwertermittlung in einer Nachsteuerrechnung

4.1 Konzeption, Aufbau und Anwendung eines phasen- und geschäftsbereichsdifferenzierenden Nachsteuermodells

4.2 Analyse und Anwendung von Nachsteuermodellen bei gewinnorientierter Ausschüttung im eingeschwungenen Zustand

4.3 Würdigung des erweiterten Nachsteuermodells aus theoretischer und bewertungspraktischer Sicht

5. Zusammenfassung und Ausblick

Anhang

A. Finanzierungstheoretische und modellbezogene Herleitungen

B. Mathematischer Anhang

C. Übersichten und Berechnungen zu den Fallstudien

D. Formelübersichten 
Inhaltsverzeichnis

Anhangsverzeichnis

Abkürzungsverzeichnis

Verzeichnis der Symbole, Indizes und Zeichen

Abbildungsverzeichnis

Tabellenverzeichnis

1. Einleitung

1.1 Problemstellungen und Zielsetzungen

I. Theoretische Grundlagen

2. Theoretische Grundlagen der kapitalmarktorientierten Unternehmensbewertung

2.1 Bewertungsmethodische, konzeptionelle und steuerliche Grundlagen

2.1.1 Phasenmethodik

2.1.2 Ableitung der finanziellen Überschüsse und Abgrenzung der Ausschüttungspolitiken

2.1.3 Überblick über wesentliche Kennzahlen bei der Cashflow-Prognose

2.1.4 Charakterisierung des Steuersystems

2.2 Finanzierungstheoretische Grundlagen und Bestimmung von Kapitalkostensätzen

2.2.1 Charakterisierung des Kapitalmarktes und Abgrenzung der Finanzierungsannahmen

2.2.2 Bestimmung von Fremdkapitalkostensätzen 75

2.2.3 Bestimmung von Eigenkapitalkostensätzen 
2.3 Bewertung mittels des Free Cashflow (FCF) und des Flow to Equity (FtE) Verfahrens sowie des Residualgewinn-Ansatzes

2.3.1 Systematisierung der Discounted Cashflow Verfahren

2.3.2 Konzeption und Aufbau des FCF Verfahrens

2.3.2.1 Vorsteuerrechnung

2.3.2.2 Modifizierte Nachsteuerrechnung

2.3.3 Konzeption und Aufbau des FtE Verfahrens

2.3.3.1 Vorsteuerrechnung

2.3.3.2 (Modifizierte) Nachsteuerrechnung

2.3.4 Der Residualgewinn-Ansatz in einer Vorsteuerrechnung

2.4 Determinanten des Wachstums in der Restwertphase und Marktwertimplikationen

3. Restwertermittlung in einer Vorsteuerrechnung

3.1 Kritische Reflexion von Vorsteuermodellen und

Erweiterungspotenziale für die Restwertmodellierung

3.1.1 Systematisierung der Modelle zur Wertermittlung in der Grobplanungshase

3.1.2 Modelle zur disaggregierten Wertermittlung in der Rentenphase

3.1.2.1 Modell nach Koller et al. (2015)

3.1.2.2 Modell nach Daves et al. (2004)

3.1.2.3 Modell nach Meitner (2013)

3.1.2.4 Modell nach Dierkes/Schäfer (2017) und Implikationen für ein erweitertes Restwertmodell

3.2 Konzeption, Aufbau und Anwendung eines phasen- und geschäftsbereichsdifferenzierenden Vorsteuermodells

3.2.2 Modellaufbau und Analyse von Modellspezifikationen 173

3.2.2.1 Restwertermittlung ohne Berücksichtigung von zusätzlichen Nettoinvestitionen 
3.2.2.2 Ermittlung der Restwertsteigerung infolge zusätzlicher Nettoinvestitionen

3.2.2.2.1 FCF Verfahren

3.2.2.2.2 FtE Verfahren

3.2.2.3 Ermittlung des Restwertes des verschuldeten Unternehmens

3.2.2.4 Spezialfall: Konstante Gesamtausschüttungsquote des investierenden Bereichs

3.2.3 Anwendung des Vorsteuermodells in Fallstudien

3.2.3.1 Charakterisierung der Basisvarianten des erweiterten Restwertmodells

3.2.3.2 Restwertermittlung ohne Berücksichtigung von zusätzlichen Nettoinvestitionen

3.2.3.3 Ermittlung der Restwertsteigerungen und des Restwertes des Unternehmens

3.3 Konzeption, Aufbau und Anwendung der Restwertermittlung bei gewinnorientierter Ausschüttung im eingeschwungenen Zustand

3.3.1 Objektivierung des Ausschüttungsverhaltens

3.3.2.1 Restwertermittlung bei kapitalwerterhöhender Verzinsung der zusätzlichen Thesaurierungen

3.3.2.2 Restwertermittlung bei kapitalwertneutraler Verzinsung der zusätzlichen Thesaurierungen

3.4 Würdigung des erweiterten Vorsteuermodells aus theoretischer und bewertungspraktischer Sicht 
4. Restwertermittlung in einer Nachsteuerrechnung

4.1 Konzeption, Aufbau und Anwendung eines phasen- und geschäftsbereichsdifferenzierenden Nachsteuermodells

4.1.1 Rekurs auf die Modellkonzeption aus der

Vorsteuerrechnung

4.1.2 Modellaufbau und Analyse von Modellspezifikationen 245

4.1.2.1 Restwertermittlung ohne Berücksichtigung von zusätzlichen Nettoinvestitionen

4.1.2.1.1 FCF Verfahren

4.1.2.1.2 FtE Verfahren

4.1.2.2 Ermittlung der Restwertsteigerung infolge zusätzlicher Nettoinvestitionen

4.1.2.2.1 FtE Verfahren

4.1.2.2.2 FCF Verfahren

4.1.2.3 Ermittlung des Restwertes des verschuldeten Unternehmens

4.1.2.4 Spezialproblem: Verzinsungssätze bei Kapitalwertneutralität

4.1.3 Anwendung des Nachsteuermodells in Fallstudien

4.1.3.1 Restwertermittlung ohne Berücksichtigung von zusätzlichen Nettoinvestitionen

4.1.3.2 Ermittlung der Restwertsteigerungen und des Restwertes des Unternehmens

270

4.1.3.2.1 Fallstudie 1b

4.1.3.2.2 Fallstudie $2 b$

4.2 Analyse und Anwendung von Nachsteuermodellen bei gewinnorientierter Ausschüttung im eingeschwungenen Zustand

4.2.1 Steuer- und ausschüttungspolitisches Kalkül sowie Systematisierung der Nachsteuermodelle

4.2.2 Konzeption, Aufbau und Beurteilung der kapitalwertneutralen Modelle nach Tschöpel et

al. (2010) und Pawelzik (2010)

4.2.2.1 Modell nach Tschöpel et al. (2010)

4.2.2.2 Modell nach Pawelzik (2010)

4.2.2.3 Beurteilung der Modelle

4.2.3 Modellerweiterung nach Meitner (2008) 
4.2.4 Konzeption, Aufbau und Anwendung eines Modells mit abgestimmter Ausschüttungs- und Finanzierungspolitik

4.2.4.1 Objektivierte Restwertermittlung auf der Basis des Modells nach Diedrich et al. (2018)

4.2.4.2 Fallstudie 3b

4.2.4.2.1 Restwertermittlung bei kapitalwerterhöhender Verzinsung der zusätzlichen Thesaurierungen

4.2.4.2.2 Restwertermittlung bei kapitalwertneutraler Verzinsung der zusätzlichen Thesaurierungen

4.3 Würdigung des erweiterten Nachsteuermodells aus theoretischer und bewertungspraktischer Sicht

5. Zusammenfassung und Ausblick

Anhang

A. Finanzierungstheoretische und modellbezogene Herleitungen

B. Mathematischer Anhang

C. Übersichten und Berechnungen zu den Fallstudien

D. Formelübersichten

Literaturverzeichnis

Stichwortverzeichnis 
https://doi.org/10.5771/9783845292120-1

Generiert durch IP '172.22.53.54', am 26.04.2023, 14:15:54.

Das Erstellen und Weitergeben von Kopien dieses PDFs ist nicht zulässig. 
Anhangsverzeichnis

A. Finanzierungstheoretische und modellbezogene Herleitungen

A.1 Anpassungsformeln für Kapitalkostensätze bei wertabhängiger Finanzierung

A.1.1 Vorsteuersätze bei residualer Ausschüttung

A.1.2 Nachsteuersätze bei residualer Ausschüttung

A.1.3 Zur Irrelevanz der Ausschüttungspolitik in einer Vorsteuerrechnung bei kapitalwertneutraler zusätzlicher Thesaurierung

A.1.4 Vereinfachter Ansatz zur Herleitung ausschüttungsabhängiger Nachsteuersätze bei kapitalwertneutraler zusätzlicher Thesaurierung

A.1.5 Nachsteuerzahlungen und Zirkularitäten bei der Berücksichtigung der Finanzierungseffekte in der Herleitung ausschüttungsabhängiger Nachsteuersätze

A.2 Modell nach Koller et al. (2015)

A.2.1 Grenzwertanalysen

A.2.2 Restwertkalkül auf der Basis des ResidualgewinnAnsatzes

A.2.3 Restwertkalkül auf der Basis des FCF Verfahrens

A.3 Restwertkalkül nach Daves et al. (2004) auf der Basis des Residualgewinn-Ansatzes

A.4 Modell nach Meitner (2013)

A.4.1 Ermittlung der Gesamtwachstumsrate des zusätzlichen Cashflows

A.4.2 Restwertkalkül auf der Basis des FCF Verfahrens $\quad 370$

A.5 Modell nach Dierkes/Schäfer (2017)

A.5.1 Ermittlung des zusätzlichen NOPLAT

A.5.2 Ermittlung der Bewertungsgrößen auf Unternehmensebene 
A.5.3 Grenzwertanalysen

A.5.4 Restwertkalkül auf der Basis des FCF Verfahrens

A.6 Vorsteuerkalkül zur Ermittlung der Restwertsteigerung bei kapitalwerterhöhender zusätzlicher Thesaurierung

B. Mathematischer Anhang

B.1 Mathematische Grundlagen

B.2 Mathematische Umformungen zu Kapitel 2

B.2.1 Ableitung der nominalen Wachstumsrate (2.10)

B.2.2 Umformungen zu Formel (2.71b)

400

B.2.3 Umformungen zu Formel (2.72)

400

B.3 Mathematische Umformungen zu Kapitel 3

401

B.3.1 Umformungen zu Formel (A.98)

401

B.3.2 Differenzbildung zwischen (3.123) und (3.128)

403

B.3.3 Differenzbildung zwischen (3.119) und (3.123)

405

B.3.4 Differenzbildung zwischen (3.119) und (3.128)

405

B.3.5 Differenzbildung zur Erläuterung von (3.137)

406

B.4 Mathematische Umformungen zu Kapitel 4

407

B.4.1 Ableitung von (4.32)

407

B.4.2 Ableitung von (4.85a)

410

B.4.3 Äquivalenz von (4.85a) und (4.85b)

411

B.4.4 Nachweis der Inflations- und Steuerneutralität von (4.87)

B.4.5 Äquivalenz von (4.87a) und (4.87b)

B.4.6 Ableitung von (4.82) und (4.88)

413

B.4.7 Umformungen zu Formel (4.95)

414

B.4.8 Umformungen zu Formel (4.98b)

414

C. Übersichten und Berechnungen zu den Fallstudien 416

C.1 Fallstudien zur Vorsteuerrechnung

C.1.1 Tabellen und Berechnungen zu den Fallstudien 1a und $2 \mathrm{a}$

C.1.2 Tabellen zur Fallstudie 3a 
C.2 Fallstudien zur Nachsteuerrechnung

C.2.1 Tabellen und Berechnungen zu den Fallstudien 1b und $2 b$

C.2.2 Tabellen zur Fallstudie 3b

D. Formelübersichten

503

D.1 Allgemeine Formeln

503

D.2 Formeln zur Anpassung der Kapitalkostensätze an den Verschuldungsgrad bei wertabhängiger Finanzierung

D.2.1 Vorsteuerrechnung

504

D.2.2 Nachsteuerrechnung 505

D.3 Restwertkalküle

508

D.3.1 Gliederung

508

D.3.2 Vorsteuerrechnung

509

D.3.3 Nachsteuerrechnung

518

D.3.4 Systematisierung der Spezialfälle nach

Dierkes /Schäfer (2017)

530 
https://doi.org/10.5771/9783845292120-1

Generiert durch IP '172.22.53.54', am 26.04.2023, 14:15:54.

Das Erstellen und Weitergeben von Kopien dieses PDFs ist nicht zulässig. 
Abkürzungsverzeichnis

\begin{tabular}{|c|c|}
\hline Abb. & Abbildung \\
\hline Anm. & Anmerkung \\
\hline APV & Adjusted Present Value \\
\hline Aufl. & Auflage \\
\hline bzw. & beziehungsweise \\
\hline CAPM & Capital Asset Pricing Model \\
\hline CF biz & Corporate Finance biz (Zeitschrift) \\
\hline d. h. & das heißt \\
\hline DCF & Discounted Cashflow \\
\hline DIH & $\begin{array}{l}\text { Days Inventory Held (Vorratsdauer, Lagerbestandsreich- } \\
\text { weite) }\end{array}$ \\
\hline DPO & $\begin{array}{l}\text { Days Payables Outstanding (Kreditorenlaufzeit, Verbind- } \\
\text { lichkeitenreichweite) }\end{array}$ \\
\hline DSO & $\begin{array}{l}\text { Days Sales Outstanding (Debitorenlaufzeit, Forderungs- } \\
\text { reichweite) }\end{array}$ \\
\hline e. V. & eingetragener Verein \\
\hline ebd. & ebenda \\
\hline EBIT & $\begin{array}{l}\text { Earnings Before Interest and Taxes (Gewinn vor Zinsen } \\
\text { und Steuern; operatives Ergebnis) }\end{array}$ \\
\hline EBITDA & $\begin{array}{l}\text { Earnings Before Interest, Taxes, Depreciation and Amorti- } \\
\text { zation (Gewinn vor Zinsen, Steuern, Abschreibungen und } \\
\text { sonstigen Finanzierungsaufwendungen) }\end{array}$ \\
\hline edn. & edition (Auflage) \\
\hline EStG & Einkommensteuergesetz \\
\hline et al. & und andere (lat. et alii) (Autoren) \\
\hline f. & folgende (Seite) \\
\hline
\end{tabular}


FAUB Fachausschuss für Unternehmensbewertung und Betriebswirtschaft (des IDW)

FB Finanz-Betrieb (Zeitschrift)

FCF Free Cashflow

ff. folgende (Seiten)

Fn. Fußnote

$\mathrm{FtE}$

Flow to Equity

GE

Geldeinheiten

$\mathrm{GuV}$

Gewinn- und Verlustrechnung (en)

Hrsg.

Herausgeber

http

Hypertext Transfer Protocol

i. d. F.

in der Fassung

i. V.m.

in Verbindung mit

IDW

Institut der Wirtschaftsprüfer in Deutschland e. V.

IDW ES

Entwurf eines IDW Standards

IDW S

IDW Standard

Kap.

Kapitel

LuL

Lieferungen und Leistungen

m. w. N.

mit weiteren Nennungen / Nachweisen

MVA

Market Value Added (Marktwertbeitrag)

n.a.

nicht angegeben, nicht verfügbar (not available)

n.F. neue Fassung

NOPLAT

Net Operating Profit Less Adjusted Taxes

RNOA

Return on Net Operating Assets

ROCE

Return on Capital Employed

ROE

Return on Equity

ROIC

Return on Invested Capital

RONIC

Return on New Invested Capital

ROTIC

Return on Total Invested Capital

S.

Seite (n) 
SolZ

SSRN

Tab.

TCF

Tz.

u. a.

URL

Verf.

$\mathrm{Vgl}$.

WACC

WPg

WPH

z. B.
Solidaritätszuschlag

Social Science Research Network

Tabelle

Total Cashflow

Teilziffer

und andere (Orte)

Uniform Resource Locator

Verfasserin

Vergleiche

Weighted Average Cost of Capital

Die Wirtschaftsprüfung (Zeitschrift)

Wirtschaftsprüferhandbuch (Edition 2018)

zum Beispiel 
https://doi.org/10.5771/9783845292120-1

Generiert durch IP '172.22.53.54', am 26.04.2023, 14:15:54.

Das Erstellen und Weitergeben von Kopien dieses PDFs ist nicht zulässig. 
Verzeichnis der Symbole, Indizes und Zeichen

Bei Symbolen und Indizes mit zwei Bedeutungen ist die häufiger verwendete Bedeutung an erster Stelle genannt.
A. Symbole

\section{A.1 Symbole des lateinischen Alphabets}

$\begin{array}{ll}B C F & \text { Basiscashflow } \\ d & \text { (1) Nenner (denominator) } \\ & \text { (2) Erwartete (r) Dividendenrendite, Dividendenanteil } \\ D & \text { Marktwert des Fremdkapitals (Market Value Debt) und } \\ & \text { Buchwert des Fremdkapitals (Book Value Debt) } \\ D i v & \text { Dividende (Dividend) } \\ E & \text { Marktwert des Eigenkapitals (Market Value Equity) } \\ F C F & \text { Freier Cashflow (Free Cashflow) } \\ F T E & \text { Flow to Equity } \\ g & \text { Reale Wachstumsrate } \\ I & \text { Fremdkapitalzinsen (Interest) } \\ I C & \text { Gesamtkapitalbestand (Invested Capital) } \\ I E & \text { Eigenkapitalbestand (Invested Equity), Buchwert des Ei- } \\ & \text { genkapitals (Book Value Equity) } \\ I R R & \text { Ökonomische Reinvestitionsrendite (Internal Rate of Re- } \\ & \text { turn) } \\ k & \text { (1) Gesamtkapitalkostensatz (Gewichteter/gewogener } \\ & \text { durchschnittlicher Kapitalkostensatz) } \\ & \text { (2) Erwartete (r, s) Kursrendite, Kurssteigerungsanteil, } \\ & \text { Kurswachstum } \\ k d & \text { Fremdkapitalkostensatz (Cost of Debt) }\end{array}$


ke

$L$

MVA

$n$

NI

NOPLAT

$O P$

$q$

$r$

$R E$

$R I$

$R O E$

ROIC

RONIC

ROTIC

$S$

$S$

$T$

$T S$

V

w

$x$
Eigenkapitalkostensatz (Cost of Equity)

Verschuldungsgrad (Leverage, Gearing)

Marktwertbeitrag (Market Value Added)

(1) Nettoinvestitionsrate (Net Investment Rate)

(2) Anzahl der intermediären Zeitpunkte einer Investitionsfolge

Nettoinvestitionen (Net Investments)

Jahresüberschuss des unverschuldeten Unternehmens oder -bereichs (Net Operating Profit Less Adjusted Taxes)

Jahresüberschuss des verschuldeten Unternehmens oder -bereichs (Operating Profit)

Ausschüttungsquote

Risikoloser Zinssatz (Riskless Rate of Return)

Thesaurierung (Retained Earnings)

Residualgewinn (Residual Income)

Eigenkapitalrendite (Return on Equity)

Rendite des investierten Kapitals (Return on Invested Capital)

Rendite des Zusatzkapitals (Return on New Invested Capital)

Gesamtkapitalrendite (Return on Total Invested Capital)

(1) Persönlicher Steuersatz (Personal Tax Rate)

(2) Investitionsfolge

Menge von Investitionsfolgen

Unternehmensteuern (Corporate Taxes)

Tax Shield (Fremdfinanzierungsbedingter Steuervorteil)

(1) Restwert (Continuing Value, Terminal Value)

(2) Marktwert (Market Value)

Nominale Wachstumsrate

Zahlung 
A.2 Symbole des griechischen Alphabets

$\begin{array}{ll}\alpha & \text { Konvergenzfaktor, Widerstandsfähigkeit (,Persistence“); } \\ \beta & 0<\alpha<1 \\ \Delta & \text { Betafaktor } \\ \Theta & \text { Veränderung } \\ \pi & \text { Fremdkapitalquote } \\ & \text { (Unternehmensspezifische) inflationsbedingte Wachstums- } \\ \tau & \text { rate } \\ & \text { (1) Unternehmensteuersatz (Corporate Tax Rate) } \\ & \text { (2) Teilsteuersatz (als Solidaritätszuschlagssatz) }\end{array}$

B. Indizes zur Spezifikation der Symbole

B.1 Indizes des lateinischen Alphabets

a

A

$\overline{\mathrm{A}}$

$\mathrm{ab}$

$\mathrm{AB}$

ac
(1) auf den Operating Profit der Anlage A bezogen (als Index der Ausschüttungsquote $q$ oder der Nettoinvestitionsrate $n$ )

(2) die Abgeltungsteuer betreffend (als Index des persönlichen Steuersatzes $s$ )

des Unternehmensbereichs A bzw. des Kernbereichs des Unternehmensbereichs A bzw. des Kernbereichs nach Abzug von Zusatzinvestitionen

auf den Flow to Equity der Anlage A bezogen (im Zusammenhang mit der Bemessung des zu investierenden Anteils in die Anlage B)

auf den freien Cashflow der Anlage A bezogen (im Zusammenhang mit der Bemessung des zu investierenden Anteils in die Anlage B)

auf den Flow to Equity der Anlage A bezogen (im Zusammenhang mit der Bemessung des zu investierenden Anteils in die Anlage C) 
$\mathrm{AC}$

$\mathrm{ad}$

$\mathrm{AD}$

b

B

bc

BC

$\mathrm{BCF}$

$\mathrm{c}$

$\mathrm{C}$

$\mathrm{cg}$

cp

$\mathrm{cR}$

d

D

Dav auf den freien Cashflow der Anlage A bezogen (im Zusammenhang mit der Bemessung des zu investierenden Anteils in die Anlage C)

auf den Flow to Equity der Anlage A bezogen (im Zusammenhang mit der Bemessung des zu investierenden Anteils in die Anlage D)

auf den freien Cashflow der Anlage A bezogen (im Zusammenhang mit der Bemessung des zu investierenden Anteils in die Anlage D)

auf den Operating Profit der Anlage B bezogen

des Unternehmensbereichs B bzw. des Zusatzbereichs

auf den Flow to Equity der Anlage B bezogen (im Zusammenhang mit der Bemessung des zu investierenden Anteils in die Anlage C)

auf den freien Cashflow der Anlage B bezogen (im Zusammenhang mit der Bemessung des zu investierenden Anteils in die Anlage C)

auf den Basiscashflow bezogen

auf den Operating Profit der Anlage $\mathrm{C}$ bezogen

des Unternehmensbereichs C bzw. des Zusatzbereichs

mit einer periodenunabhängigen Gesamtwachstumsrate (,,constant growth policy“)

mit einer bereichseinheitlichen periodenunabhängigen Ausschüttungsquote (,,constant payout policy“)

mit einer bereichseinheitlichen periodenunabhängigen Reinvestitionsrendite (,constant Return on Invested Capital (ROIC)“)

(1) auf Dividenden bezogen (als Index des persönlichen Steuersatzes $s$ )

(2) auf den Operating Profit der Anlage D bezogen (als Index der Ausschüttungsquote $q$ oder der Nettoinvestitionsrate $n$ )

der Anlage D

nach Daves et al. (2004) und Ehrhardt (2005a, b) 
$D S$

$f$

FCF

$\mathrm{FtE}$

g

$G S$

$\mathrm{k}$

$\mathrm{KN}$

KNB

$\mathrm{KNz}$

$\mathrm{Kol}$

$\ell$

$\mathrm{Mei}$

$\mathrm{OP}$

$\mathrm{P}$

q

$\mathrm{qB}$

$\mathrm{r}$

Ref

$\mathrm{S}$

SZ

$t$

$\mathrm{u}$

Z

nach Dierkes /Schäfer (2017)

Fremdkapitalkategorie; $f=1, \ldots, F$

auf den freien Cashflow bezogen

auf den Flow to Equity bezogen

(1) auf Kursgewinne bezogen (als Index des persönlichen Steuersatzes $s$ )

(2) für reales Wachstum

gesamt

nach Gordon/Shapiro (1956)

auf das Kernkapital bezogen

Kapitalwertneutralität auf Unternehmensebene

Kapitalwertneutralität in dem Geschäftsbereich B

Kapitalwertneutralität der Investition in die Zusatzanlage nach Koller et al. (2015)

des verschuldeten Unternehmens oder -bereichs nach Meitner (2008, 2013)

auf den Operating Profit bezogen

„Praktikermethode“ nach Pawelzik (2010) und Tschöpel et al. $(2010 a, b)$

(1) das Ausschüttungsverhalten berücksichtigend

(2) bei gewinnorientierter Ausschüttung

Identität der Ausschüttungsquoten $q_{\mathrm{II}}^{\mathrm{AB}}$ und $q_{\mathrm{II}}^{\mathrm{B}}$

bei residualer Ausschüttung

der Referenzanlage

nach persönlichen Steuern

auf den Solidaritätszuschlag bezogen

Periode oder Zeitpunkt des mehrphasigen Prognosezeitraums einschließlich Detailprognosephase;

$t=0, \ldots, T_{\mathrm{I}}, \ldots, T_{\mathrm{II}}, \ldots,+\infty$

des unverschuldeten Unternehmens oder -bereichs auf das Zusatzkapital bezogen 
Periode oder Zeitpunkt der Bemessung zusätzlicher Investitionen; $z=1, \ldots,+\infty ; z_{\mathrm{I}}=1, \ldots, \Phi_{\mathrm{I}} ; z_{\mathrm{II}}=1, \ldots,+\infty$

B.2 Indizes des griechischen Alphabets

$\theta$

$\kappa$

$\lambda$

$v$

$\pi$

$\tau$

$\phi$

B.3 Indizes des römischen Alphabets

Informationsstand (B, C, D)) lich investiert wird) Tax Shields

Bereichsindex; $\kappa=\{\mathrm{A}, \mathrm{B}, \mathrm{C}, \mathrm{D}\}$ (der Bereich, der zusätzlich investiert $(\mathrm{A}, \mathrm{B})$ oder in den zusätzlich investiert wird

Bereichsindex; $\lambda=\{B, C, D\}$ (der Bereich, in den zusätz-

Laufparameter für Periode oder Zeitpunkt

inflationsbedingt (-getrieben, -induziert)

unter Berücksichtigung des unternehmensteuerbedingten

Periode oder Zeitpunkt einer Prognosephase;

$\phi=1, \ldots,+\infty ; \phi_{\mathrm{I}}=1, \ldots, \Phi_{\mathrm{I}} ; \phi_{\mathrm{II}}=1, \ldots,+\infty$

C. Zeichen

C.1 Spezielle mathematische Zeichen

Kennzeichnung als Zufallsvariable

$\mathrm{E}[\cdot] \quad$ Erwartungswertoperator

$\forall$

„für alle“

$\epsilon$

„Element von“ 
$[a ; b)$

(rechtsseitig) halboffenes Intervall mit der eingeschlossenen Untergrenze $a$ und der nicht-eingeschlossenen Obergrenze $b$

$\infty$

Unendlich

$\mathbb{Z}$

Menge der ganzen Zahlen; $\{0 ; \pm 1 ; \pm 2 ; \pm 3 ; \ldots\}$

$\cup$

Vereinigung disjunkter Teilmengen

usw., logische Fortsetzung

C.2 Seitenwechsel anzeigende Zeichen

$\neg \quad$ „Fortsetzung auf der Folgeseite“, „Fortsetzung unten“

( $\neg$, „mit Fortsetzung auf der/den Folgeseite(n)“, ,mit Fortsetzung unten“"

„Ende der Fortsetzung“ 
https://doi.org/10.5771/9783845292120-1

Generiert durch IP '172.22.53.54', am 26.04.2023, 14:15:54.

Das Erstellen und Weitergeben von Kopien dieses PDFs ist nicht zulässig. 
Abbildungsverzeichnis

- Kapitel 2 -

2-1 Gliederung des Prognosezeitraums in Phasen, Perioden und Zeitpunkte

2-2 Systematisierung der Discounted Cashflow Verfahren

- Kapitel 3 -

3-1 Wachstumshypothesen in den Prognosephasen eines DreiPhasen-Modells (Teil 1)

(a) Abrupte Senkung der konstanten Wachstumsrate zum Zeitpunkt $T_{\text {II }}$

(b) Linearer Rückgang der Wachstumsrate zwischen $T_{\mathrm{I}}$ und $T_{\text {II }}$

3-2 Wachstumshypothesen in den Prognosephasen eines Konvergenzmodells und eines Drei-Phasen-Modells (Teil 2)

(a) Konvergierende Wachstumsrate ab dem Zeitpunkt $T$

(b) Konvergierende Wachstumsrate zwischen $T_{\mathrm{I}}$ und $T_{\mathrm{II}}$

3-3 Schematisierung der Entwicklung der Bewertungsgrößen nach dem Modell von Koller et al. (2015) in den ersten drei Perioden der Rentenphase ( $\neg$ )

3-4 Schematisierung der Zusammenhänge zwischen Kernkapital, Basiscashflow und Zusatzinvestitionen nach dem Modell von Meitner (2013) bis zur zweiten Periode der Rentenphase

3-5 Schematisierung der Weiterentwicklung der periodischen Zusatzinvestitionen nach dem Modell von Meitner (2013) bis zur dritten Periode der Rentenphase 
3-6 Schematisierung der Entwicklung der Bewertungsgrößen nach dem Modell von Dierkes /Schäfer (2017) in den ersten drei Perioden der Rentenphase $(\neg)$

3-7 Allgemeine Darstellung der Kapitalisierung aller zusätzlichen freien Cashflows in der Restwertphase nach dem erweiterten Modell

A-1 Schematisierung der Weiterentwicklung der ersten Zusatzinvestition nach dem Modell von Meitner (2013) in den vier Folgeperioden $(\neg)$ 
Tabellenverzeichnis

- Kapitel 2 -

2-1 Abgrenzung der Prognosephasen 56

2-2 Wesentliche Kennzahlen bei der Aufstellung der Plan-Bilanz 69

2-3 Wesentliche Kennzahlen bei der Aufstellung der Plan-GuV 70

- Kapitel 3 -

3-1 Nettoinvestitionen nach dem Modell von Koller et al. (2015) in den ersten drei Perioden der Rentenphase und Ermittlung des Gesamtkapitals zum Zeitpunkt $\phi_{\mathrm{II}}=3$

3-2 Entwicklung der NOPLAT nach dem Modell von Koller et al. (2015) und Ermittlung der gesamten NOPLAT in den ersten drei Perioden der Rentenphase

3-3 Entwicklung der freien Cashflows nach dem Modell von Koller et al. (2015) und Ermittlung der gesamten freien Cashflows in den ersten drei Perioden der Rentenphase

3-4a Entwicklung des Gesamtkapitals und des Kernkapitals nach dem Modell von Daves et al. (2004) in den ersten drei Perioden der Rentenphase

3-4b Entwicklung des Zusatzkapitals nach dem Modell von $D a$ ves et al. (2004) in den ersten drei Perioden der Rentenphase

3-5 Alternative Investitionsbeziehungen in den Fallstudien 1 und 2

3-6 Steuersatz und Fremdkapitalkostensatz des Unternehmens sowie bereichsspezifische Eigenkapitalkostensätze bei reiner Eigenfinanzierung 
3-7a Bereichsspezifische Nettoinvestitionsraten, ROIC und Fremdkapitalquoten zu Beginn der Grobplanungsphase und zugehörige Konvergenzfaktoren

3-7b Bereichsspezifische Nettoinvestitionsraten, ROIC und Fremdkapitalquoten in der Rentenphase

3-8 Wachstumsraten in der Rentenphase

3-9 Fallstudie 1a / 1b: Prognostizierte Entwicklung des in den Bereich C investierten Anteils am freien Cashflow des Kernbereichs im Restwertzeitraum

3-10 Fallstudie 1a / 1b: Prognostizierte Entwicklung des in den Bereich $\mathrm{C}$ investierten Anteils am freien Cashflow des Bereichs B im Restwertzeitraum

3-11 Fallstudie 2a / 2b: Prognostizierte Entwicklungen der in die Bereiche $\mathrm{B}$ und $\mathrm{C}$ investierten Anteile am freien Cashflow des Kernbereichs im Restwertzeitraum

3-12 Fallstudie 2a / 2b: Prognostizierte Entwicklung des in den Bereich $\mathrm{C}$ investierten Anteils am verbleibenden freien Cashflow des Kernbereichs im Restwertzeitraum

3-13 Fallstudie 2a: Prognostizierte Entwicklungen des ROIC und der Rentabilitätsspanne im Bereich $\mathrm{C}$ bei Kapitalwertneutralität in der Rentenphase

3-14 Fallstudie 3a /3b: Steuersatz, Verschuldungsgrad, Fremdkapitalkostensatz und objektivierte Ausschüttungsquote des Unternehmens

3-15 Fallstudie 3a / 3b: Nettoinvestitionsrate, ROIC, Wachstumsrate und Kapitalkostensätze des Kernbereichs in einer Vorsteuerrechnung

3-16 Fallstudie 3a/3b: Ausschüttungsquote, ROE, Wachstumsrate und Kapitalkostensätze der Zusatzanlage in einer Vorsteuerrechnung 
4-1 Fallstudie 1b/2b: Steuersätze

4-2 Fallstudie 1b/2b: Fremdkapitalkostensatz des Unternehmens sowie bereichsspezifische Eigenkapitalkostensätze bei reiner Eigenfinanzierung

4-3 Fallstudie 2b: Prognostizierte Entwicklung des ROIC im Bereich $\mathrm{C}$ bei Kapitalwertneutralität in der Rentenphase

4-4 Fallstudie 3b: Steuersätze

4-5 Fallstudie 3b: Fremdkapitalkostensatz des Unternehmens

4-6 Fallstudie 3b: Kapitalkostensätze des Kernbereichs in einer Nachsteuerrechnung

4-7 Fallstudie 3b: Kapitalkostensätze der Zusatzanlage in einer Nachsteuerrechnung

\section{- Anhang $\mathrm{C}-$}

C-1 Fallstudie 1/2: Prognostizierte Entwicklungen der bereichsspezifischen Nettoinvestitionsraten, ROIC und Fremdkapitalquoten im Restwertzeitraum $(\neg)$

C-2 Wertetabelle für den Kernbereich

C-3 Wertetabelle für den Bereich B ( $\neg)$

C-4 Wertetabelle für den Bereich C

C-5 Fallstudie 1a/2a: Restwertermittlung im Kernbereich mit dem FCF und dem FtE Verfahren ohne Berücksichtigung der zusätzlichen Nettoinvestitionen ( $\neg$ )

C-6 Fallstudie 1a/2a: Restwertermittlung im Bereich B mit dem FCF und dem FtE Verfahren ohne Berücksichtigung der zusätzlichen Nettoinvestitionen ( $\neg$ )

C-7 Fallstudie 1a/2a: Restwertermittlung im Bereich C mit dem FCF und dem FtE Verfahren bei kapitalwerterhöhender Verzinsung des Invested Capital in der Rentenphase ( $\neg)$ 
C-8 Fallstudie 1a: Ermittlung der Restwertsteigerung aufgrund der zusätzlichen Nettoinvestitionen des Kernbereichs in den Bereich C mit dem FCF und dem FtE Verfahren $(\neg)$

C-9 Fallstudie 1a: Ermittlung der Marktwerte der zusätzlichen Nettoinvestitionen des Kernbereichs in den Bereich $\mathrm{C}$ mit dem FCF Verfahren $(\neg)$

C-10 Fallstudie 1a: Ermittlung der Restwertsteigerung aufgrund der zusätzlichen Nettoinvestitionen des Bereichs B in den Bereich C mit dem FCF und dem FtE Verfahren ( $\neg)$

C-11 Fallstudie 1a: Ermittlung der Marktwerte der zusätzlichen Nettoinvestitionen des Bereichs B in den Bereich C mit dem FCF Verfahren $(\neg)$

C-12 Fallstudie 1a: Bereichsspezifische ROE, Thesaurierungsquoten und Gesamtausschüttungsquoten im Restwertzeitraum

C-13 Fallstudie 1a: Aggregation der Bewertungsgrößen im Bereich $\mathrm{C}$ aufgrund der zusätzlichen Nettoinvestitionen vom Kernbereich

C-14 Fallstudie 1a: Aggregation der Bewertungsgrößen im Bereich $\mathrm{C}$ aufgrund der zusätzlichen Nettoinvestitionen von Bereich B

C-15 Fallstudie 1a: Aggregation der Bewertungsgrößen auf Unternehmensebene $(\neg)$

C-16 Fallstudie 2a: Ermittlung der Restwertsteigerung aufgrund der zusätzlichen Nettoinvestitionen des Kernbereichs in den Bereich B mit dem FCF und dem FtE Verfahren ( $\neg)$

C-17 Fallstudie 2a: Ermittlung der Restwertsteigerung aufgrund der zusätzlichen Nettoinvestitionen des Kernbereichs in den Bereich $\mathrm{C}$ mit dem FCF und dem FtE Verfahren bei kapitalwerterhöhender Verzinsung des Invested Capital in der Rentenphase ( $\neg)$

C-18 Fallstudie 2a: Aggregation der Bewertungsgrößen im Bereich $B$ aufgrund der zusätzlichen Nettoinvestitionen vom Kernbereich 
C-19 Fallstudie 2a: Aggregation der Bewertungsgrößen im Bereich $\mathrm{C}$ aufgrund der zusätzlichen Nettoinvestitionen vom Kernbereich

C-20 Fallstudie 2a: Aggregation der Bewertungsgrößen auf Unternehmensebene $(\neg)$

C-21 Fallstudie 2a: Restwertermittlung im Bereich C mit dem FCF und dem FtE Verfahren bei kapitalwertneutraler Verzinsung des Invested Capital in der Rentenphase ( $\neg$ )

C-22 Fallstudie 2a: Ermittlung der Restwertsteigerung aufgrund der zusätzlichen Nettoinvestitionen des Kernbereichs in den Bereich $\mathrm{C}$ mit dem FCF und dem FtE Verfahren bei kapitalwertneutraler Verzinsung des Invested Capital in der Rentenphase ( $\neg)$

C-23 Fallstudie 3a: Restwertermittlung im Kernbereich bei residualer Ausschüttung mit dem FCF und dem FtE Verfahren

C-24 Fallstudie 3a: Ermittlung der Restwertsteigerung aufgrund der zusätzlichen Nettoinvestitionen des Kernbereichs mit dem FCF und dem FtE Verfahren

C-25 Fallstudie 3a: Ermittlung der Marktwerte der zusätzlichen Nettoinvestitionen mit dem FtE und dem FCF Verfahren

C-26 Fallstudie 3a: Aggregation der Bewertungsgrößen der Zusatzanlage D aufgrund der zusätzlichen Nettoinvestitionen vom Kernbereich

C-27 Fallstudie 3a: Aggregation der Bewertungsgrößen auf Unternehmensebene

C-28 Fallstudie 1b/2b: Kapitalkostensätze und modifizierter Steuersatz des Kernbereichs in einer Nachsteuerrechnung $(\neg)$

C-29 Fallstudie 1b/2b: Kapitalkostensätze und modifizierter Steuersatz des Bereichs B in einer Nachsteuerrechnung

C-30 Fallstudie 1b/2b: Kapitalkostensätze und modifizierter Steuersatz des Bereichs $\mathrm{C}$ in einer Nachsteuerrechnung

C-31 Fallstudie 1b/2b: Restwertermittlung im Kernbereich mit dem FCF und dem FtE Verfahren ohne Berücksichtigung der zusätzlichen Nettoinvestitionen $(\neg)$ 
C-32 Fallstudie 1b/2b: Restwertermittlung im Bereich B mit dem FCF und dem FtE Verfahren ohne Berücksichtigung der zusätzlichen Nettoinvestitionen ( $\neg$ )

C-33 Fallstudie 1b/2b: Restwertermittlung im Bereich C mit dem FCF und dem FtE Verfahren bei kapitalwerterhöhender Verzinsung des Invested Capital in der Rentenphase ( $\neg$ )

C-34 Fallstudie 1b: Ermittlung der Restwertsteigerung aufgrund der zusätzlichen Nettoinvestitionen des Kernbereichs in den Bereich C mit dem FCF und dem FtE Verfahren ( $\neg$ )

C-35 Fallstudie 1b: Ermittlung der Restwertsteigerung aufgrund der zusätzlichen Nettoinvestitionen des Bereichs B in den Bereich C mit dem FCF und dem FtE Verfahren $(\neg)$

C-36 Fallstudie 1b: Bereichsspezifische ROE, Thesaurierungsquoten und Gesamtausschüttungsquoten im Restwertzeitraum

C-37 Fallstudie 1b: Aggregation der Bewertungsgrößen im Bereich $\mathrm{C}$ aufgrund der zusätzlichen Thesaurierungen

C-38 Fallstudie 1b: Aggregation der Bewertungsgrößen auf Unternehmensebene $(\neg)$

C-39 Fallstudie 2b: Ermittlung der Restwertsteigerung aufgrund der zusätzlichen Nettoinvestitionen des Kernbereichs in den Bereich B mit dem FCF und dem FtE Verfahren ( $\neg)$

C-40 Fallstudie 2b: Ermittlung der Restwertsteigerung aufgrund der zusätzlichen Nettoinvestitionen des Kernbereichs in den Bereich $\mathrm{C}$ mit dem FCF und dem FtE Verfahren bei kapitalwerterhöhender Verzinsung des Invested Capital in der Rentenphase $(\neg)$

C-41 Fallstudie 2b: Aggregation der Bewertungsgrößen in den Bereichen B und C aufgrund der zusätzlichen Thesaurierungen

C-42 Fallstudie 2b: Aggregation der Bewertungsgrößen auf Unternehmensebene ( $\neg)$

C-43 Fallstudie 2b: Restwertermittlung im Bereich C mit dem FCF und dem FtE Verfahren bei kapitalwertneutraler Verzinsung des Invested Capital in der Rentenphase ( $\neg)$ 
C-44 Fallstudie 2b: Ermittlung der Restwertsteigerung aufgrund der zusätzlichen Nettoinvestitionen des Kernbereichs in den Bereich C mit dem FCF und dem FtE Verfahren bei kapitalwertneutraler Verzinsung des Invested Capital in der Rentenphase ( $\neg$ )

C-45 Fallstudie 3b: Restwertermittlung im Kernbereich bei residualer Ausschüttung mit dem FCF und dem FtE Verfahren

C-46 Fallstudie 3b: Ermittlung der Restwertsteigerung aufgrund der zusätzlichen Nettoinvestitionen des Kernbereichs mit dem FCF und dem FtE Verfahren

C-47 Fallstudie 3b: Aggregation der Bewertungsgrößen der Zusatzanlage D aufgrund der zusätzlichen Nettoinvestitionen vom Kernbereich

C-48 Fallstudie 3b: Aggregation der Bewertungsgrößen auf Unternehmensebene

D-1 Systematisierung der Spezialfälle nach Dierkes/Schäfer (2017) 
https://doi.org/10.5771/9783845292120-1

Generiert durch IP '172.22.53.54', am 26.04.2023, 14:15:54.

Das Erstellen und Weitergeben von Kopien dieses PDFs ist nicht zulässig. 